\title{
SÍNTESE, CARACTERIZAÇÃO E ESTUDO DAS PROPRIEDADES CATALIÍTICAS E MAGNÉTICAS DE NANOPARTÍCULAS DE Ni DISPERSAS EM MATRIZ MESOPOROSA DE $\mathrm{SiO}_{2}$
}

Neftalí L. V. Carreño, Edson R. Leite*, Luís P. S. Santos, Paulo N. Lisboa-Filho e Elson Longo

Centro Multidisciplinar de Desenvolvimento de Materiais Cerâmicos, Universidade Federal de São Carlos, CP 676, 13565-905

São Carlos - SP

Geórgia C. L. Araújo, Andersson Barison e Antônio G. Ferreira

Departamento de Química, Universidade Federal de São Carlos, CP 676, 13565-905 São Carlos - SP

Antoninho Valentini e Luiz Fernando D. Probst

Departamento de Química, Universidade Federal de Santa Catarina, CP 476, 88040-900 Florianópolis - SC

Recebido em $8 / 8 / 01$; aceito em $5 / 3 / 02$

\begin{abstract}
SYNTHESIS, CHARACTERIZATION AND STUDY OF MAGNETIC AND CATALYTIC PROPERTIES OF DISPERSED Ni NANOPARTICLES ON MESOPOROUS $\mathrm{SiO}_{2}$ MATRIX. Nickel nanoparticles supported on amorphous silica ceramic matrix were synthesized by the polymeric precursor method. The nanostructure was characterized by NMR, BET, XRD, SEM, TEM, and flame atomic absorption spectrometry techniques. It was observed a dependence of the crystallite size on the thermal annealing, under a $\mathrm{N}_{2}$ atmosphere. The materials presented a high catalytic activity and selectivity upon the $\beta$-pinene hydrogenation reaction. The magnetic hystereses were also correlated with the morphology of the processed material.
\end{abstract}

Keywords: nanoparticles; catalyst; magnetism.

\section{INTRODUÇ̃̃o}

Nos últimos anos o desenvolvimento de materiais nanoestruturados ganhou grande impulso nas áreas da ciência dos materiais e da química do estado sólido ${ }^{1}$. Metais de transição nanoestruturados suportados com propriedades magnéticas e catalíticas ${ }^{2}$ vêm sendo estudados, visando ao aumento da superfície específica de adsorção, da seletividade catalítica e a otimização da distribuição e do tipo de partícula magnética, com o consequiente aumento de sensibilidade, redução da temperatura de trabalho e diminuição do tempo de resposta dos dispositi$\operatorname{vos}^{2,3}$. Para isto, métodos de síntese devem ser desenvolvidos para possibilitar a obtenção de partículas nanométricas (restritamente menores que $10 \mathrm{~nm}$ ) com baixo grau de aglomeração e estreita faixa de distribuição de tamanho. Para a obtenção destes materiais destacamse os métodos dos precursores poliméricos ${ }^{4-13}$, sol-gel ${ }^{2}$ e de deposição em fase de vapor $^{10}$. Destes, o método dos precursores poliméricos (método Pechini) vem sendo usado na obtenção de pós nanométricos e filmes cerâmicos finos ${ }^{8}$. MacLachlan e colaboradores ${ }^{14}$ descrevem um interessante método de processamento de nanocompostos usando precursores poliméricos, através do qual foi possível o controle das propriedades magnéticas, direcionando-se a pirólise do material e obtendo-se partículas nanométricas de Fe dispersas em uma matriz cerâmica amorfa formada por $\mathrm{Si}, \mathrm{C}$ e $\mathrm{Ni}$.

Uma etapa crítica no processamento e manutenção de materiais nanoestruturados é o tratamento térmico. A elevada reatividade superficial das partículas facilita a sinterização produzindo, desta forma, aglomerados de tamanho macroscópico que possuem propriedades menos vantajosas que as dos compostos em escala nanométrica. Em contraste com os métodos convencionais de preparação de metais suportados, o uso de uma matriz inorgânica como hospedeira conduz à obtenção de partículas metálicas de tamanhos uniformes e dispersão homogênea de agrupamentos de átomos metálicos (“clusters"). Resinas poliméricas de troca iônica, zeólitas e refratári-

*e-mail: derl@power.ufscar.br os porosos, entre outros materiais, foram empregados por diferentes pesquisadores como matrizes hospedeiras na síntese de nanopartículas de metais de transição ${ }^{15-17}$.

Recentes publicações relatam importantes estudos sobre a cinética e o mecanismo de formação de "nanoclusters". Estes estudos podem servir como ferramentas fundamentais para a explicação de várias propriedades, tais como estabilidade física e química dos nanocompósitos $^{15,18,19}$. Dois fatores importantes no desenvolvimento de nanocompósitos que devem ser considerados, além do controle do tamanho de partícula, são os efeitos eletrostáticos (cargas em compostos inorgânicos) e os efeitos estéricos (em compostos orgânicos) na estabilização dos mesmos ${ }^{18}$.

Sabe-se que a eficiência catalítica de metais de transição sólidos freqüentemente aumenta com a diminuição do tamanho de partícula. Porém, quando partículas metálicas de tamanho reduzido são obtidas, a probabilidade de agregação dos grupos de átomos aumenta, principalmente sob tratamento térmico ${ }^{17}$. Para prevenir tal agregação, são sintetizadas freqüentemente nanopartículas metálicas absorvidas num estabilizador, o qual evita a aglomeração e ajuda no controle do tamanho da partícula ${ }^{20}$. Um exemplo disto é a síntese de nanopartículas de Pd, cataliticamente ativo, preparadas na presença de ligantes ${ }^{21}$, polímeros funcionalizados ${ }^{22,23}$ e surfactantes ${ }^{24}$.

De um modo geral, a modelagem dos poros de nanoestruturas com aplicação em processos catalíticos precisa atingir duas metas essenciais: a) gerar uma porosidade nas matrizes hospedeiras que permita o acesso dos reagentes aos centros reativos, o que pode ser alcançado por uma área superficial específica elevada e mesoporosidade; b) assegurar a acessibilidade dos centros ativos metálicos com microporosidade ${ }^{25}$.

As propriedades magnéticas dos materiais dependem de fatores intrínsecos e da dimensionalidade, isto é, da forma como estes se apresentam, por exemplo nanocristais (tridimensional) e filmes finos (bidimensional). Nos casos em que as amostras não são volumosas, uma ou mais das suas dimensões podem ter grandeza macroscópica ou nanoscópica. Os efeitos da redução nas dimensões 
do material sobre as propriedades magnéticas surgem quando as dimensões da amostra se aproximam do comprimento de intercâmbio (em escala atômica $)^{26}$.

Os "clusters" de interesse para o magnetismo são usualmente formados de partículas metálicas magnéticas dispersas numa matriz não-magnética ou compósitos de material magnético, sendo que os mesmos podem apresentar aplicações para gravações magnéticas. Dependendo do tamanho, os "clusters" apresentam diferentes comportamentos magnéticos, em particular, mostram diferentes mecanismos para a reversão da direção de magnetização sob efeito de um campo magnético externo ${ }^{27,28}$.

Para partículas magnéticas muito pequenas, a direção de magnetização pode flutuar com a excitação térmica; neste caso as partículas apresentam um comportamento superparamagnético. Esta denominação é dada pelo fato destes sistemas apresentarem propriedades análogas às dos paramagnéticos, com momento magnético efetivo intensificado ${ }^{26}$.

\section{Precursores poliméricos (Método Pechini)}

O método Pechini consiste na formação de quelatos entre os cátions metálicos (dissolvidos em solução aquosa) com ácidos carboxílicos (ácido cítrico) e posterior polimerização através de uma reação de poliesterificação com poliálcoois (etilenoglicol), visando uma distribuição melhor dos cátions (aleatoriedade) a nível atômico, na estrutura polimérica.

$\mathrm{O}$ aquecimento a temperaturas moderadas $\left(100\right.$ a $\left.200^{\circ} \mathrm{C}\right)$, sob atmosfera de ar ou outros gases, causa reações de condensação, com a formação de moléculas de água e de ésteres. Durante o aquecimento a estas temperaturas ocorre a poliesterificação e a maior parte do excesso de água é removida, resultando uma "resina" polimérica sólida, que pode ser dissolvida em água, dependendo da relação estequiométrica dos reagentes. Após este estágio, o poliéster é decomposto em $\mathrm{CO}_{2}$ e água, a aproximadamente $300^{\circ} \mathrm{C}$, para eliminar o excesso de material orgânico. Estudos realizados por Zanetti ${ }^{29}$ têm demonstrado que, variando-se a relação ácido cítrico/etilenoglicol, observa-se somente variações nas temperaturas de eliminação do material orgânico. Outras reações não são observadas.

A relação ácido cítrico/metal é outro fator importante na estequiometria dos óxidos. Chu e Dunn ${ }^{30}$ obtiveram óxidos supercondutores de $\mathrm{YBa}_{2} \mathrm{Cu}_{3} \mathrm{O}_{7}$ pelo processo de citrato amorfo usando uma relação ácido cítrico/metal igual a um equivalente-grama, sendo esta a quantidade mínima de ácido cítrico necessária para coordenar todos os íons metálicos.

As diversas técnicas de síntese de partículas metálicas encontradas na literatura conduzem à obtenção de materiais alternativos com diferentes propriedades catalíticas e/ou magnéticas. Sendo assim, alguns dos parâmetros mais importantes a serem observados na escolha do método de síntese são aqueles que se direcionam a um melhor controle de área superficial, menor tamanho de partículas e baixo grau de aglomeração.

Neste trabalho é apresentada a síntese de nanopartículas de $\mathrm{Ni}$ metálico numa matriz cerâmica de $\mathrm{SiO}_{2}$ mesoporosa, assim como a resposta do material processado no estudo de magnetização (histerese) à temperatura ambiente e à atividade catalítica na reação de hidrogenação seletiva do terpeno $\beta$-pineno ${ }^{31}$.

\section{PARTE EXPERIMENTAL}

\section{Obtenção dos precursores poliméricos}

O citrato metálico foi obtido através da simples dissolução de 0,672 moles de ácido cítrico (Merck) em 100 mL de etanol (Synth) sob agitação mecânica. Após a completa dissolução do ácido cítrico, foram adicionados $50 \mathrm{~mL}$ de tetraetoxisilano (TEOS) da Aldrich, seguido do acréscimo de nitrato de níquel hexahidratado (Merck). Para a obtenção da resina polimerizada foram adicionados $62,17 \mathrm{~mL}$ de etilenoglicol (Aldrich).

As diferentes amostras foram preparadas seguindo-se o procedimento descrito acima, variando-se a estequiometria entre TEOS e nitrato de níquel hexaidratado (conforme o percentual de níquel desejado). A relação molar de 3/1 entre ácido cítrico e TEOS e a relação mássica de 6/4 entre ácido cítrico e etilenoglicol foram mantidas constantes. As amostras sintetizadas foram designadas como $X-\mathrm{SiO}_{2}$, onde o $X$ corresponde ao percentual de $\mathrm{Ni}$ em massa por grama de amostra.

\section{Tratamento térmico}

Após a preparação dos precursores poliméricos, as resinas foram ligeiramente aquecidas numa chapa de aquecimento, com a finalidade de aumentar a viscosidade das mesmas e, em seguida, levadas a uma mufla termoprogramada na temperatura de $225^{\circ} \mathrm{C}$, por um período de $4 \mathrm{~h}$. O polímero pirolisado ("puff”), material obtido após esse tratamento das resinas, foi desagregado por moagem num moinho tipo atritor (Szegvari Attritor System 01HD) para a obtenção de um pó com granulometria menor que 300 mesh. Em seguida, o material foi calcinado em um forno lacrado EDG, tipo tubo, e tratado termicamente a diferentes temperaturas entre 300 a $800^{\circ} \mathrm{C}$, com tempos variáveis e com taxa de aquecimento de $12{ }^{\circ} \mathrm{C} / \mathrm{min}$ sob atmosfera protetora de $\mathrm{N}_{2}$, com fluxo de $1 \mathrm{~cm}^{3} / \mathrm{min}$, sendo posteriormente resfriado até $50{ }^{\circ} \mathrm{C}$ a uma taxa de $8{ }^{\circ} \mathrm{C} / \mathrm{min}$.

\section{Instrumentação}

Para a caracterização por difração de raios-X, foi utilizado um difratômetro Siemens D5000, usando a radiação $\mathrm{K} \alpha$ do $\mathrm{Cu}$ e monocromador de grafite. A área superficial específica foi obtida utilizando-se o equipamento ASAP 2000 da Micromeritics. O teor metálico nas amostras foi determinado por espectrometria de absorção atômica de chama (FAAS), num equipamento Varian AA-800, equipado com uma lâmpada de cátodo ôco operando a $351,5 \mathrm{~nm}$ e corrente de 4 $\mathrm{mA}$, resolução do monocromador de $0,2 \mathrm{~nm}$ e chama ar-acetileno em condição oxidante. As análises elementares foram realizadas no equipamento CHNS-O FISONS EA 1108. Espectros de RMN de ${ }^{29} \mathrm{Si}$ das soluções obtidas da dissolução de ácido cítrico em etanol e adicionadas de TEOS foram adquiridos num espectrômetro BRUKER, modelo DRX400, 9,4 Tesla (operando em 79,49 MHz na frequiência do ${ }^{29} \mathrm{Si}$ ) com a finalidade de verificar os tipos de interações ocorridas entre TEOS e ácido cítrico. Gotas de DMSO- $d_{6}$ foram adicionadas para o ajuste da homogeneidade do campo magnético. A sequiência de pulsos utilizada para obtenção dos espectros foi a "zg30", a qual emprega pulsos de $30^{\circ}$ com duração de $4 \mu \mathrm{s}$, reciclos de $5 \mathrm{~s}$ e tempo de aquisição de $0.7 \mathrm{~s}$ (Janela espectral de $47.000 \mathrm{~Hz}$ ).

Para a caracterização microestrutural foram usados os microscópios eletrônicos de varredura (Zeiss, DSM940A) e de transmissão (CM200 Philips Holland, 200 kV). Na análise dos produtos obtidos no teste catalítico foi utilizado um cromatógrafo gasoso SHIMADZU GC-14B, com coluna CBP1 equipado com detetor de ionização de chama. As medidas de magnetização (histerese) foram realizadas à temperatura ambiente no magnetômetro SQUID Quantum Design MPMS-5 usando campos magnéticos aplicados de até 1,0 Tesla.

\section{RESULTADOS E DISCUSSÃO}

\section{Citrato metálico}

O procedimento experimental descrito neste trabalho permite a 
obtenção de um citrato metálico a partir do uso de um agente quelante (ácido cítrico). Inicialmente, é promovida a formação de um complexo metálico entre TEOS e ácido cítrico dissolvidos em etanol. Posteriormente, o complexo metálico inclui também o sal de níquel sem que seja ocasionada a hidrólise total do TEOS. Os espectros de RMN de ${ }^{29} \mathrm{Si}$ demonstram a ocorrência da reação de complexação (Si-citrato) devido ao surgimento de novos sinais quando se adiciona ácido cítrico ao TEOS (Figura 1). Na Figura 1a, antes da adição de ácido cítrico, observa-se somente os sinais em $\delta$ 0,0 e 81,9 ppm referentes aos deslocamentos químicos do TMS (tetrametilsilano, referência interna) e do TEOS, respectivamente. Na Figura 1b, além dos sinais mencionados, também são observados outros sinais atribuídos aos complexos formados entre ácido cítrico e TEOS. O mesmo é observado quando são comparados os espectros de RMN de ${ }^{1} \mathrm{H}$ e ${ }^{13} \mathrm{C}$, não mostrados aqui, antes e depois da reação, demonstrando inequivocamente a ocorrência da formação de complexos. Isto é corroborado por trabalhos realizados por Sjoblom ${ }^{32}$ e Nijdam ${ }^{33}$ em estudos de ressonância magnética nuclear de organosilanos, os quais utilizam TEOS como precursor.

As intensas absorções no intervalo de $\delta-70$ a -130 ppm, observadas em ambos os espectros de ${ }^{29} \mathrm{Si}$ (Figura 1), referem-se aos núcleos de silício contidos no tubo de vidro e em outras partes da sonda do espectrômetro de RMN.

Os dados obtidos através de espectrometria de ressonância magnética nuclear de ${ }^{13} \mathrm{C},{ }^{1} \mathrm{H}$ e de ${ }^{29} \mathrm{Si}$ também sugerem que a relação molar ideal de 1/3 de TEOS e ácido cítrico, respectivamente, permite a formação e estabilidade do citrato metálico (organosilano).

A Figura 2 descreve o modelo proposto para obtenção do citrato metálico (TEOS, sal de níquel e ácido cítrico dissolvidos em etanol) e a posterior formação do precursor polimérico, usando etilenoglicol.

A natureza da associação e a estrutura formada nos diversos
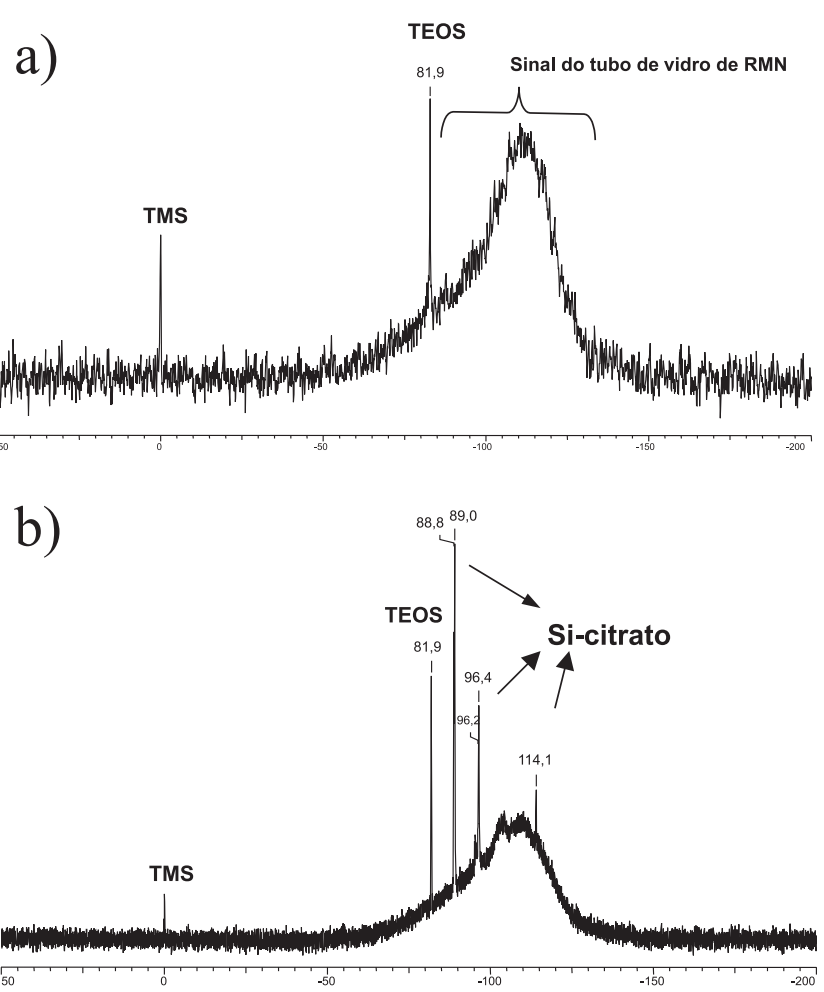

Figura 1. Espectros de ressonância magnética nuclear de ${ }^{29} \mathrm{Si}$, do reagente de partida TEOS (a) e do complexo metálico (Si-citrato) TEOS-ácido cítrico (b), usando o TMS como referência

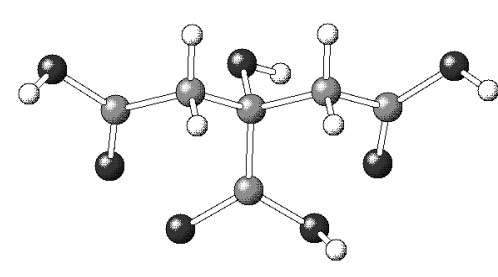

Ácido cítrico

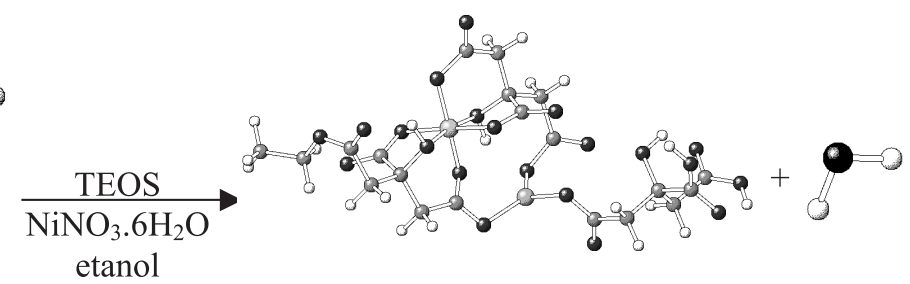

etanol

Citrato metálico de Si e Ni

Água

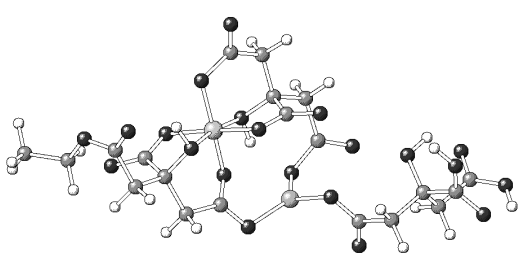

Citrato metálico de $\mathrm{Si}$ e Ni

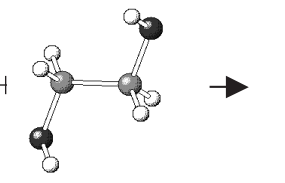

Etileno glicol<smiles>O=S(=O)=O</smiles>

Polímero

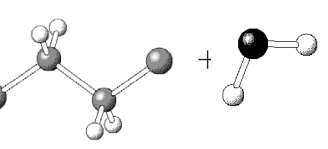

Água

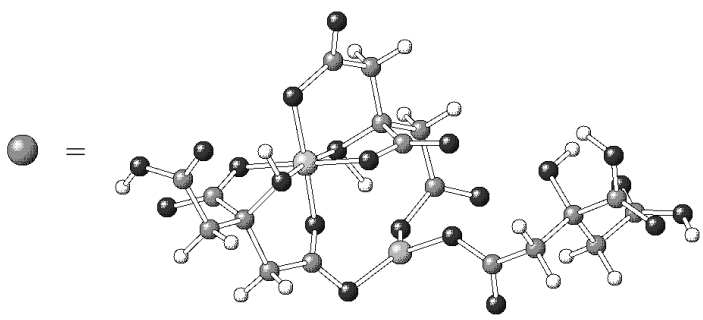

Legenda

Carbono

Hidrogênio

Silício

Níquel

Oxigênio

Figura 2. Modelo molecular proposto para a o processo de formação do citrato metálico e o precursor polimérico 
citratos metálicos estão em estudo e serão descritas em trabalhos futuros.

\section{Tratamento térmico}

O pré-tratamento térmico a que são submetidos os precursores poliméricos tem como objetivo eliminar a maior parte do material orgânico. Um segundo tratamento térmico em atmosfera inerte $\left(\mathrm{N}_{2}\right)$ permite a obtenção de nanopartículas de Ni dispersas em sílica em uma única etapa. Isto ocorre devido à geração de $\mathrm{CO}$ promovida pela decomposição térmica do material orgânico remanescente, o qual atua como agente redutor do sal de Ni. Com base na análise elementar (CHNS-O), Figura 3, observou-se que, mesmo após o tratamento térmico sob atmosfera inerte, uma quantidade significativa de material orgânico (carbono) não foi eliminada durante o tratamento térmico. A eliminação do material orgânico presente nas amostras indica a possibilidade da formação de defeitos, fato estreitamente relacionado com a porosidade do material. Boury ${ }^{25} \mathrm{e} \mathrm{Shea}^{34}$ apresentaram trabalhos indicando a viabilidade do desenvolvimento de moléculas orgânicas e inorgânicas de materiais híbridos, com a finalidade de modelar e controlar a porosidade das estruturas à base de organosilanos.

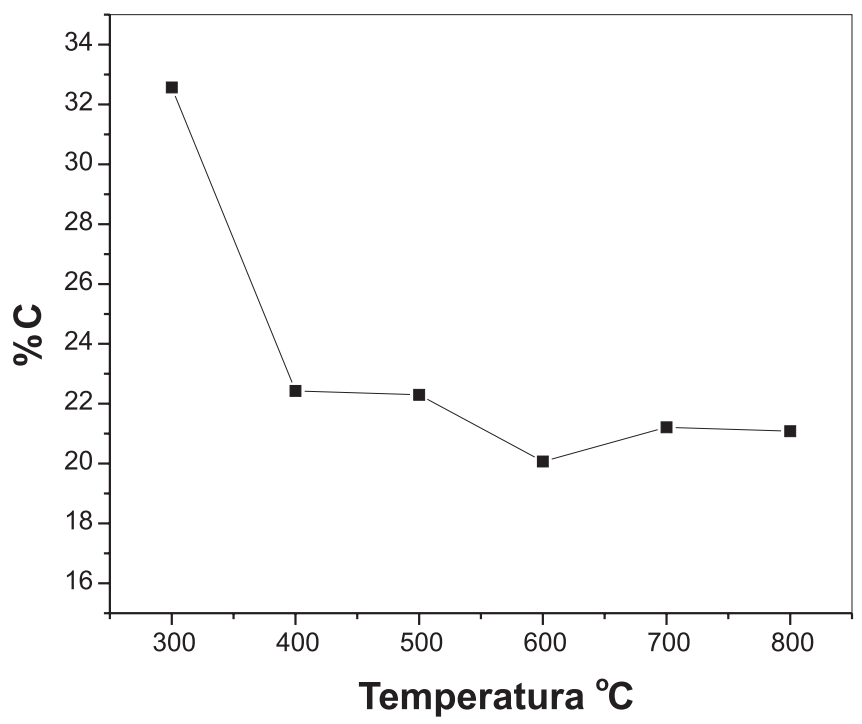

Figura 3. Perda de carbono em função da temperatura de tratamento térmico da amostra 12,5- $\mathrm{SiO}_{2}$ sob atmosfera inerte de $\mathrm{N}_{2}$, acompanhada por análise elementar (CHN)

\section{Nanopartículas dispersas em sílica amorfa}

A geração de Ni metálico no processo de calcinação é comprovada pelos resultados de difração de raios-X, obtidos após o tratamento térmico das amostras em atmosfera de $\mathrm{N}_{2}$ sem a necessidade de uma atmosfera redutora $\left(\mathrm{H}_{2}\right)$. Os difratogramas típicos das nanopartículas de Ni dispersas em uma matriz de sílica amorfa, tratadas em temperaturas que variaram de 225 a $800^{\circ} \mathrm{C}$, apresentaram uma fase cristalina correspondente ao níquel metálico e uma fase amorfa da matriz de sílica (Figura 4). Os picos com valor de $2 \theta$ igual a 44,5 (plano cristalino 111) e 51,8 (plano cristalino 200) encontrados nos difratogramas mostram a presença de partículas cristalinas de Ni metálico ${ }^{35}$.

O tamanho médio do cristalito de Ni nas amostras obtidas após o tratamento térmico em atmosfera inerte foi caracterizado por difração de raios-X, utilizando-se a largura a meia altura (FWHM) dos difratogramas das amostras (DRX), por meio da equação de Scherrer $^{35,36}$.

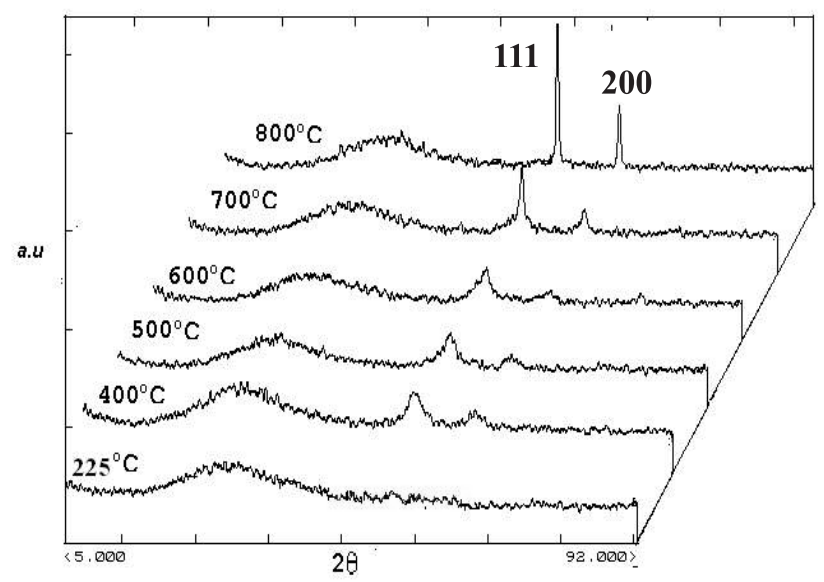

Figura 4. Difratogramas de raios-X das nanopartículas de Ni dispersas em uma matriz amorfa, obtidos em diferentes temperaturas de tratamento térmico durante $2 \mathrm{~h}$ sob atmosfera inerte de $\mathrm{N}_{2}$, da amostra $12,5-\mathrm{SiO}_{2}$

$$
D_{\mathrm{SCH}}=\mathrm{k} \lambda /\left\{\left[\left(\beta_{\mathrm{obs}}\right)^{2}-\left(\beta_{\mathrm{p}}\right)^{2}\right]^{1 / 2} \cos \theta\right\}
$$

Onde $D_{\mathrm{SCH}}$ é o tamanho do cristalito, $\lambda$ é o comprimento de onda $(0,15405 \mathrm{~nm}), \mathrm{k}$ é a constante igual a $1, \theta$ é ângulo de difração do plano cristalino, $\beta_{\text {obs }}$ é a largura a meia altura para o pico de intensidade de $100 \%$ [plano (111)] das amostras e $\beta_{\mathrm{p}}$ é a largura a meia altura do pico de difração do padrão externo $\left(\mathrm{SiO}_{2}\right)$. Neste estudo, o perfil do pico de difração foi ajustado usando-se a função pseudoVoigth para calcular FWHM.

O tamanho médio de cristalito e os respectivos teores de Ni (determinados por espectrometria de absorção atômica) em cada amostra estão apresentados na Tabela 1 . As amostras tratadas a $500{ }^{\circ} \mathrm{C}$ por $2 \mathrm{~h}$ apresentaram tamanho médio de cristalito de Ni próximo de $4 \mathrm{~nm}$.

Tabela 1. Tamanho de cristalito das partículas de níquel para as diferentes razões entre a $\mathrm{SiO}_{2} / \mathrm{Ni}$ e diferentes temperaturas de tratamento térmico (durante $2 \mathrm{~h}$ )

\begin{tabular}{ccc}
\hline \% de $\mathrm{Ni}^{-\mathrm{SiO}_{2}}$ & $\begin{array}{c}\text { Temperatura de } \\
\text { tratamento térmico } \\
\left({ }^{\circ} \mathrm{C}\right)\end{array}$ & $\begin{array}{c}\text { Tamanho de cristalito (nm) } \\
\text { (considerando o } \\
\text { plano cristalino } \\
(111) \text { do Ni) }\end{array}$ \\
\hline $47-\mathrm{SiO}_{2}$ & 500 & 3,8 \\
$23-\mathrm{SiO}_{2}$ & 500 & 3,4 \\
$12,5-\mathrm{SiO}_{2}$ & 500 & 5,2 \\
$10-\mathrm{SiO}_{2}$ & 500 & 4,8 \\
$8,7-\mathrm{SiO}_{2}$ & 500 & 4,6 \\
$3-\mathrm{SiO}_{2}$ & 500 & 4,7 \\
$5-\mathrm{SiO}_{2}$ & 400 & 4,6 \\
$5-\mathrm{SiO}_{2}$ & 500 & 4,7 \\
$5-\mathrm{SiO}_{2}$ & 600 & 4,6 \\
$5-\mathrm{SiO}_{2}$ & 700 & 13,6 \\
$5-\mathrm{SiO}_{2}$ & 800 & 41,3 \\
\hline
\end{tabular}

O estudo da morfologia típica das amostras à base de sílica foi acompanhado por microscopia eletrônica de varredura de elétrons secundários (MEV). Os resultados mostraram partículas com formas alongadas, como ilustrado na Figura 5. 


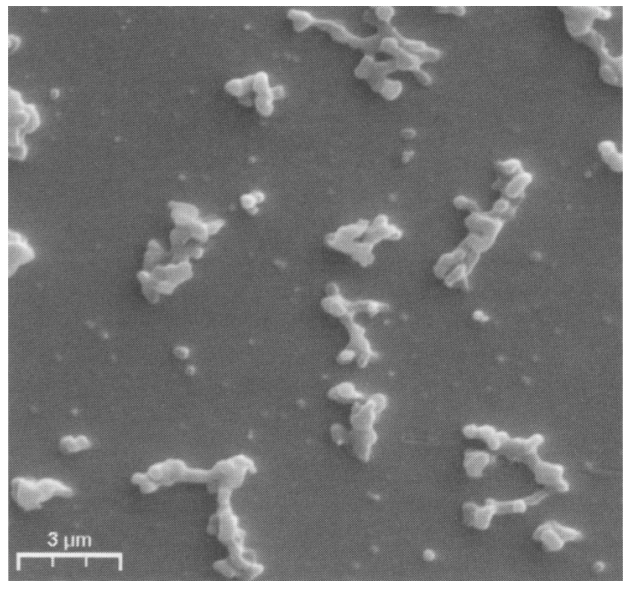

Figura 5. Imagens obtidas através de microscopia eletrônica de varredura (MEV), típica de um nanocompósito à base de sílica amorfa

A Figura 6a ilustra imagens de campo claro obtidas por microscopia eletrônica de transmissão (TEM) da amostra contendo $23 \%$ em massa de Ni, termicamente tratada em atmosfera inerte. Os pontos escuros, homogeneamente dispersos no interior de uma estrutura tipicamente amorfa ${ }^{37,38}$ (sílica), observados na imagem de TEM, correspondem às nanopartículas de Ni. A figura 6a (inserida) ilustra o padrão de difração de elétrons típicos de um policristal de Ni metálico em escala nanométrica ${ }^{37}$ dispersos em sílica amorfa (anéis grossos e pouco intensos) da amostra $23-\mathrm{SiO}_{2}$. A diminuição da quantidade de Ni no precursor polimérico promove aumento na dispersão metálica na matriz amorfa, como pode ser observado na imagem de campo claro da amostra $3-\mathrm{SiO}_{2}$ (Figura 6b). Os dados obtidos por TEM corroboram com os valores de tamanho médio de cristalitos calculados por DRX, apresentados na Tabela 1.
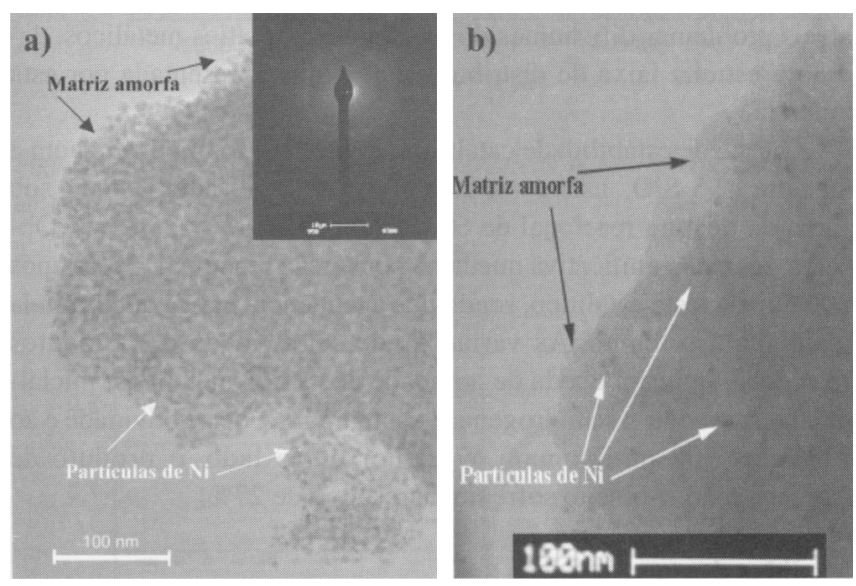

Figura 6. Imagens de campo claro de microscopia eletrônica de transmissão (TEM), das amostras tratadas a $500{ }^{\circ} \mathrm{C}$ durante $2 \mathrm{~h}$ : a) $23-\mathrm{SiO}$, padrão de difração de elétron (Figura inserida) e b) $3-\mathrm{SiO}_{2}$

A análise de adsorção-dessorção de $\mathrm{N}_{2}$ sugere microporosidade (Figura 7a) para concentrações acima de $5 \%$ em massa de Ni. As curvas de adsorção-dessorção de $\mathrm{N}_{2}$ indicaram uma mesoporosidade no material, ilustrada na Figura $7 \mathrm{~b}$. As curvas de distribuição de tamanho de poros obtidas pelo método de Barret-Joyner-Halenda $(\mathrm{BJH})^{39}$ para as amostras $5-\mathrm{SiO}_{2}$ e $47-\mathrm{SiO}_{2}$, termicamente tratadas a $500{ }^{\circ} \mathrm{C}$ por $2 \mathrm{~h}$, são apresentadas na figura 8 . A Figura 8 a mostra que há uma estreita faixa de distribuição de poros (30 ̊) para a amostra 5- $\mathrm{SiO}_{2}$. Com o incremento da concentração de Ni há uma distribuição bi-modal muito clara do tamanho dos poros na matriz amorfa, Figuras $8 \mathrm{~b}$ e $8 \mathrm{c}$ das amostras $12,5-\mathrm{SiO}_{2}$ e $47-\mathrm{SiO}_{2}$, respectivamente.

A amostra com menor concentração de $\mathrm{Ni}\left(5-\mathrm{SiO}_{2}\right)$ possui uma área superficial específica $\left(\mathrm{S}_{\mathrm{BET}}\right)$ de $280 \mathrm{~m}^{2} / \mathrm{g}$, enquanto que os materiais com maior concentração de $\mathrm{Ni}\left(12,5-\mathrm{SiO}_{2}\right.$ e $\left.47-\mathrm{SiO}_{2}\right)$ apresentaram áreas superficiais específicas menores $\left(\mathrm{S}_{\mathrm{BET}}\right)$ de $247 \mathrm{~m}^{2} / \mathrm{g}$ e $202 \mathrm{~m}^{2} / \mathrm{g}$, respectivamente. Este fato pode ser explicado como resultado do aumento da concentração de $\mathrm{Ni}$ e conseqüente preenchimento dos poros da matriz amorfa.

\section{Teste catalítico da matriz de $\mathrm{SiO}_{2} / \mathrm{Ni}$}

A avaliação da atividade catalítica das amostras obtidas foi realizada através da reação de hidrogenação seletiva do terpeno $\beta$-pineno. Para estes ensaios foram utilizadas as amostras $5-\mathrm{SiO}_{2}, 12,5-\mathrm{SiO}_{2} \mathrm{e}$ 47- $\mathrm{SiO}_{2}$. Com o objetivo de se observar a influência das diferentes amostras na razão dos produtos de hidrogenação cis e trans-pinano, foi utilizada uma massa de catalisador de $80 \mathrm{mg}$ sob fluxo de reagentes $\left(\mathrm{H}_{2}+\beta\right.$-pineno) de $40 \mathrm{~cm}^{3} / \min \left(\mathrm{WHSV}=1,4 \mathrm{~h}^{-1}\right)^{40}$. Deste modo, obteve-se a conversão total do reagente pelas amostras $12,5-\mathrm{SiO}_{2} \mathrm{e}$ $47-\mathrm{SiO}_{2}$, evitando-se assim a possível presença de produtos de isomerização tais como $\alpha$-pineno, canfeno e limoneno. Os resulta-
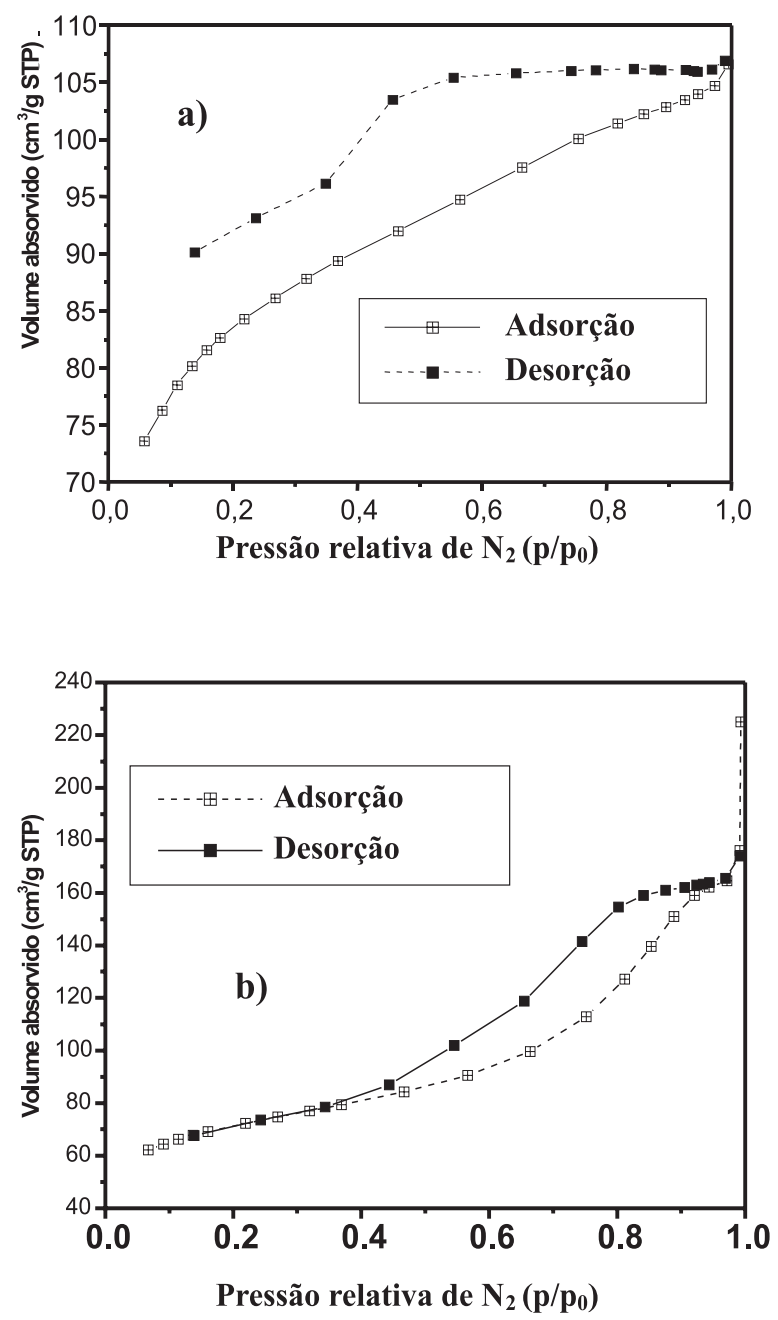

Figura 7. Adsorção-dessorção de $N_{2}$ para as amostras tratadas a $500{ }^{\circ} \mathrm{C}$ durante $2 \mathrm{~h}$ : a) 5- $\mathrm{SiO}_{2}$ e b) 47- $\mathrm{SiO}_{2}$ 


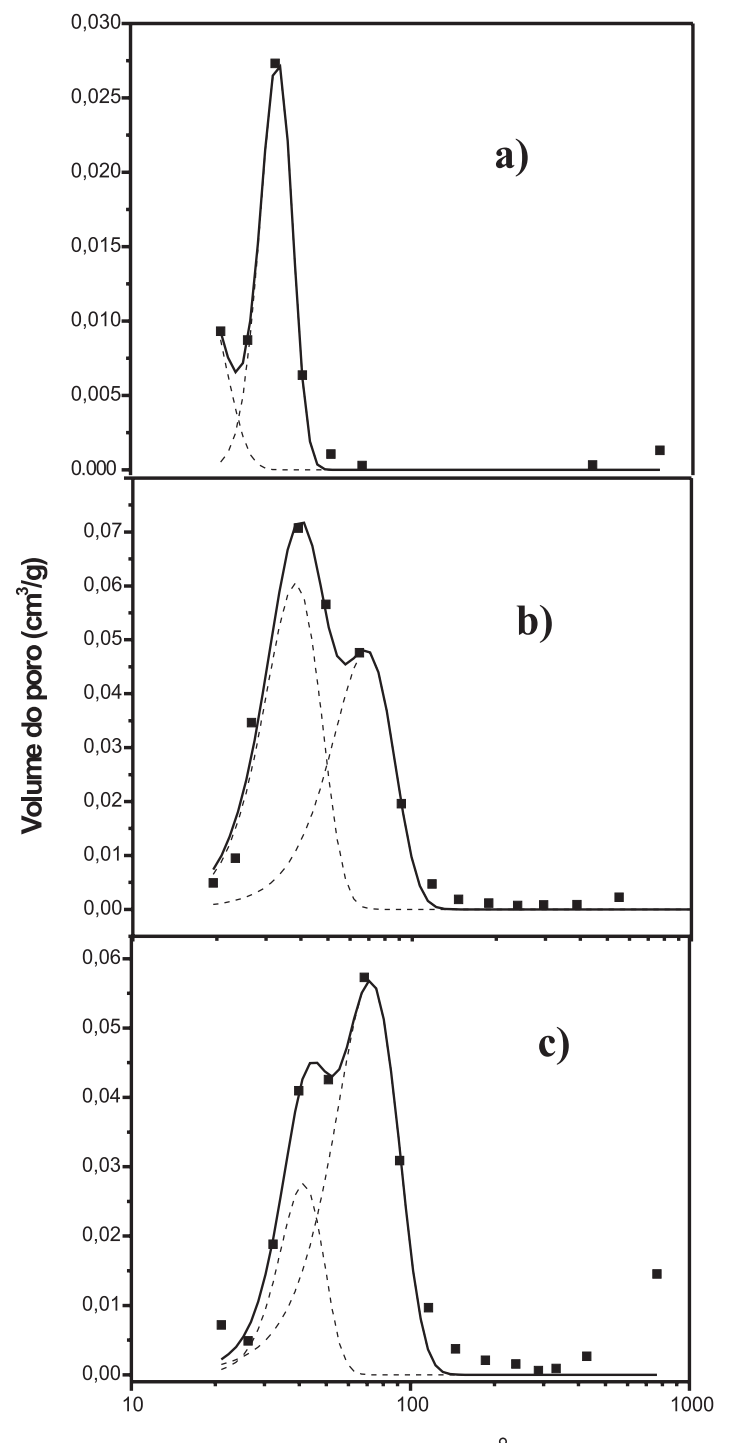

Tamanho do Poro ( $⿱$ A $)$

Figura 8. Distribuição do tamanho de poros das amostras tratadas a $500{ }^{\circ} \mathrm{C}$ durante $2 \mathrm{h:} \mathrm{a)} 5-\mathrm{SiO}_{2}$; b) 12,5- $\mathrm{SiO}_{2}$ e c) $47-\mathrm{SiO}_{2}$

dos mostram que o produto de hidrogenação cis-pinano na reação catalisada pelas amostras $12,5-\mathrm{SiO}_{2}$ e $47-\mathrm{SiO}_{2}$ é favorecido em baixas temperaturas reacionais (Figura 9), o que facilita ter um controle da razão cis/trans pinano por intermédio da temperatura reacional. Comparando-se as razões para os diferentes teores de $\mathrm{Ni}$, observa-se que a maior razão cis/trans pinano foi obtida com a amostra 47$\mathrm{SiO}_{2}$, em baixa temperatura (Figura 9).

Comportamento catalítico diferenciado foi observado para a amostra $5-\mathrm{SiO}_{2}$. Embora tenham sido utilizadas as mesmas condições experimentais (massa de catalisador e fluxo da mistura reacional de hidrogênio e $\beta$-pineno), foram obtidos somente produtos de reações de isomerização (até $90{ }^{\circ} \mathrm{C}$ ) tais como $\alpha$-pineno, canfeno e limoneno ${ }^{41}$. A conversão para produtos de hidrogenação foi muito baixa, sendo detectada somente a partir de $100{ }^{\circ} \mathrm{C}$ de temperatura reacional, atingindo $3 \%$ de cis-pinano a $120{ }^{\circ} \mathrm{C}$, com conversão total de $13 \%$ do reagente. Conforme indicado na Tabela 2, são observados elevados teores do produto de reação de isomerização ( $\alpha$-pineno). Este comportamento pode ser atribuído a possíveis sítios ácidos no material, uma vez que é conhecido o fato de que a reação de isomerização de alcenos pode ser promovida por sítios ácidos fra-

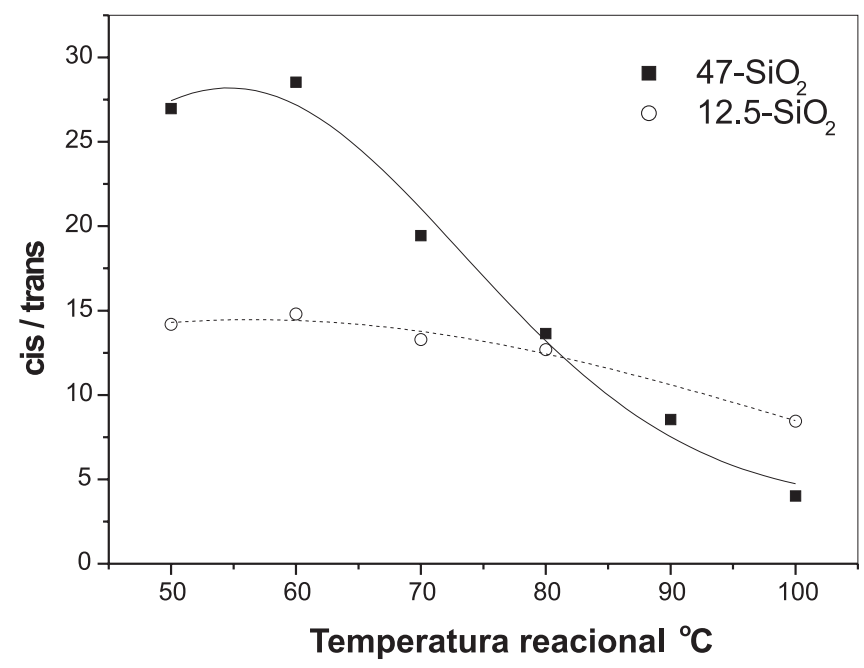

Figura 9. Razão cis-pinano/trans-pinano em função da temperatura reacional, usando-se uma massa de catalisador de $80 \mathrm{mg}$ sob um fluxo de $\mathrm{H}_{2} 40 \mathrm{~cm}^{3} / \min \left(\mathrm{WHSV}=1,4 \mathrm{~h}^{-1}\right): 47-\mathrm{SiO}_{2}$ e 12,5-SiO

Tabela 2. Valores de conversão de $\beta$-pineno para $\alpha$-pineno

\begin{tabular}{cc}
\hline Temperatura ${ }^{\circ} \mathrm{C}$ & $\% \alpha$-pineno obtido \\
\hline 50 & 0,7 \\
70 & 1,0 \\
80 & 1,5 \\
90 & 2,1 \\
100 & 3,0 \\
120 & 6,0 \\
\hline
\end{tabular}

$\cos ^{41}$. No entanto, deve-se considerar também a contribuição de possíveis problemas difusionais do reagente até os sítios metálicos, devido à estreita faixa de distribuição de poros apresentada por esta amostra.

O teste de estabilidade catalítica (Figura 10) foi realizado com a amostra 12,5- $\mathrm{SiO}_{2}$, usando-se uma massa de catalisador de $6 \mathrm{mg}$ sob fluxo da mistura reacional de $60 \mathrm{~cm}^{3} / \mathrm{min}\left(\mathrm{WHSV}=25,8 \mathrm{~h}^{-1}\right.$ ). Observa-se uma significativa queda na conversão reacional (50\%) após 500 min de teste catalítico, sendo que a tendência indica queda ainda maior com o tempo. As variações de seletividade dos produtos reacionais indicam queda de atividade dos sítios metálicos; inicialmente os produtos de hidrogenação somam $81 \%$ de seletividade e ao final dos $500 \mathrm{~min}$ somam $57 \%$. Por outro lado, o produto de isomerização $\alpha$-pineno sofre um acréscimo de $27 \%$.

\section{Medidas de magnetização}

As curvas de magnetização (histerese) em função do campo magnético foram realizadas com as amostras de $5-\mathrm{SiO}_{2}$ e $47-\mathrm{SiO}_{2}$ à temperatura ambiente e são apresentadas nas Figuras 11 e 12, respectivamente. Nas curvas, após a indução magnética no ponto $\mathbf{M}, \mathbf{o}$ material permanece magnetizado mesmo na ausência de campo magnético ( $\mathrm{H}=$ zero). Os campos coercitivos observados nas curvas de histereses das amostras $5-\mathrm{SiO}_{2}$ e $47-\mathrm{SiO}_{2}$ são de 70 Oe e 85 Oe, respectivamente. $\mathrm{O}$ tamanho do cristalito e a distância entre os mesmos estão diretamente relacionados com a resposta magnética encontrada para o presente material ${ }^{42,43}$. Os baixos valores de campos coercitivos encontrados também confirmam que as partículas de níquel 


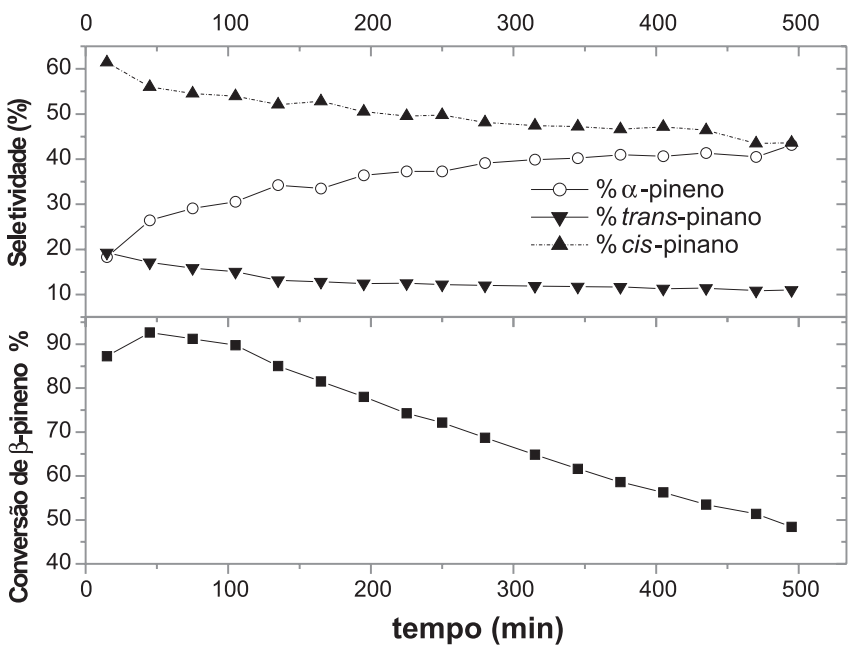

Figura 10. Reação de hidrogenação do $\beta$-pineno catalisada pela amostra 12,5-SiO ${ }_{2}$; conversão de $\beta$-pineno para cis-pinano e trans-pinano em função do tempo, $\left(T_{\text {reac̃ão }}=50^{\circ} \mathrm{C}\right)$

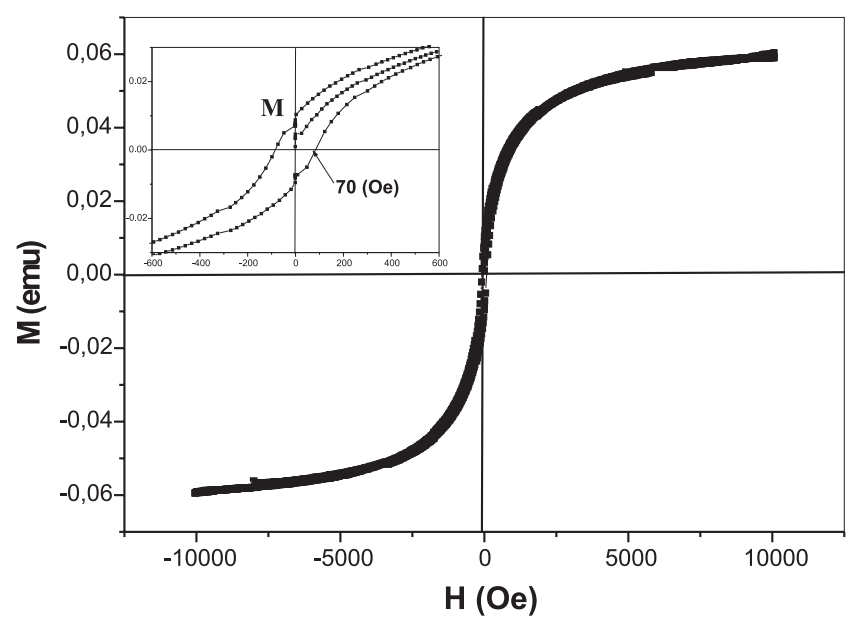

Figura 11. Histereses magnéticas características de matriz nanoestruturada de $5-\mathrm{SiO}$

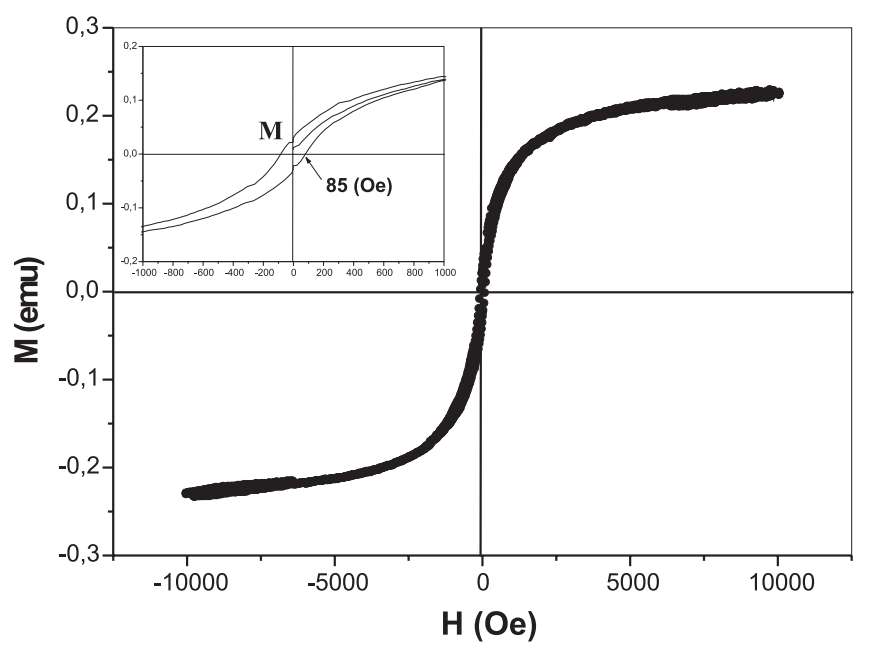

Figura 12. Histereses magnéticas características de matriz nanoestruturada de $47-\mathrm{SiO}_{2}$ metálico possuem dimensões nanométricas e sugerem baixas interações internas e externas entre os "clusters" de Ni encapsulados nos poros de sílica (diâmetro médio de aproximadamente $6 \mathrm{~nm}$ ). Não foi observada nenhuma contribuição magnética da matriz de sílica amorfa.

Nos detalhes das Figuras 11 e 12 é possível observar que os nanocompostos estão próximos do limite de um material no regime superparamagnético ${ }^{42}$, que é tipicamente definido como material com partículas muito pequenas (na ordem de nanômetros) em que não existe interação magnética entre as partículas e a direção de magnetização total da amostra, e que apresenta inversão completa da magnetização, com um tempo de relaxação muito pequeno ${ }^{27,43}$.

A modelagem e o controle do tamanho médio dos poros na matriz amorfa representam fator determinante nas possíveis aplicações catalíticas e magnéticas, uma vez que os átomos de Ni ficam alojados no interior destes poros, até a formação de uma partícula com diâmetro próximo ao dos poros do hospedeiro (sílica amorfa), o que reduz, de forma significativa, problemas de sinterização nas partículas de níquel. Os poros da sílica amorfa que hospedam as partículas metálicas (sítios catalíticos) são responsáveis pelo controle do acesso dos reagentes em fase gasosa até os sítios ativos onde ocorre o processo catalítico. Fisicamente, é necessário ressaltar que os poros da matriz amorfa funcionam como barreiras estéricas que impedem interações "interclusters" e limitam a quantidade de átomos metálicos que podem acessar um mesmo poro, diminuindo as interações "interclusters" (átomo-átomo).

De um ponto de vista geral, a modelagem estrutural dos poros hospedeiros de nanopartículas, como a síntese das mesmas, representa uma nova alternativa para a obtenção de materiais com propriedades magnéticas e catalíticas aplicáveis tecnologicamente.

\section{CONCLUSÕES}

Este trabalho mostra a síntese e caracterização físico-química de um nanocompósito formado por duas fases, uma cristalina e outra amorfa, através do método dos precursores poliméricos.

A caracterização dos compostos híbridos obtidos no tratamento térmico mostrou que o processo de formação do citrato metálico através do uso de um agente quelante (ácido cítrico), e sua posterior polimerização, permite a distribuição com homogeneidade elevada dos átomos metálicos quelados em matriz mesoporosa inorgânica. Esta última também tem um papel fundamental na estabilidade dos "clusters" metálicos obtidos.

A resposta catalítica observada na reação de hidrogenação do $\beta$ pineno para as amostras testadas sugere a possibilidade de se controlar a seletividade reacional por intermédio do tamanho do cristalito, da concentração de $\mathrm{Ni}$, da distribuição e do diâmetro dos poros presentes na matriz de $\mathrm{SiO}_{2}$.

O tamanho do cristalito metálico presente na amostra influencia de forma muito significativa os resultados obtidos nas medidas magnéticas. As respostas magnéticas da matriz de sílica contendo as nanopartículas de Ni estão relacionadas diretamente com os pequenos acoplamentos entre os "clusters" de Ni.

\section{AGRADECIMENTOS}

Os autores agradecem o suporte financeiro prestado pelas agências de fomento FAPESP/CEPID, CNPq/PRONEX, FUNCITEC-SC e CAPES.

\section{REFERÊNCIAS}

1. Watson, J.; Ihokura, K.; MRS Bull. 1999, 24, 14. 
2. Poznyak, S. K.; Golubev, A.; Kulak, A. I.; Surf. Sci. 2000, 454, 396.

3. De Jongh, L. J.; Appl. Organomet. Chem. 1998, 12, 393.

4. Wang, X.; Yee, S. S.; Carey, W. P.; Sensors Actuators, B 1995, 25, 454.

5. Lisboa, P. N.; Ortiz, W. A.; Zanetti, S. M.; Leite, E. R.; J. Appl. Phys. 1999, 85,4515 .

6. Bouquet, V.; Leite, E. R.; Longo, E.; Varela, J. A.; J. Eur. Ceram. Soc. 1999, 19, 1447.

7. Zanetti, S. M.; Leite, E. R.; Longo, E.; Varela, J. A.; J. Mater. Res. 1998 , 13, 2932.

8. Leite, E. R.; Carreño, N. L. V.; Santos, L. P. S.; Rangel, J. H.; Soledade, L. E. B.; Longo, E.; Campos, C. E. M.; Lanciotti Jr., F.; Pizani, P. S.; Varela, J. A.; Appl. Phys. A 2001, 73, 567.

9. Zanetti, S. M.; Leite, E. R.; Longo, E.; Varela, J. A.; Appl. Organomet. Chem. 1999, 13, 373.

10. Champion, Y.; Bigot, J.; Nanostructur. Mater. 1998, 10, 1097.

11. Sheppard, L. M.; J. Am. Ceram. Soc. Bull. 1992, 71, 85.

12. Wright, J. S.; Francis, L. F.; Mater. Res. 1993, 8, 1712.

13. Calzada, M. L.; Del Olmo, L.; J. Non-Cryst. Solids 1990, 121, 413.

14. MacLachlan, M. J.; Ginzburg, M.; Coombs, N.; Science 2000, 287, 1460.

15. Aiken III, J. D.; Finke, R.; J. Mol. Catal. A: Chem. 1999, 145, 1.

16. Chan, Y. N. C.; Schrock, R. R.; Cohen, R. E.; Chem. Mater. 1992, 4, 205.

17. Ding, J. H.; Gin, D. L.; Chem. Mater. 2000, 12, 22.

18. Watzky, M. A.; Finke, R. G.; J. Am. Chem. Soc. 1997, 119, 10382.

19. Teranishi, T.; Miyake, M.; Chem. Mater. 1998, 10, 594.

20. Zhao, M.; Crooks, R. M.; Angew. Chem., Int. Ed. 1999, 38, 364.

21. Reetz, M. T.; Lohmer, G.; Chem. Commun. 1996, 16, 1921.

22. Teranishi, T.; Nakata, K.; Iwamoto, M.; Miyake, M.; Toshima, N.; React. Funct. Polym. 1998, 37, 111.

23. Sulman, E.; Bodrova,Y.; Platonova, O.; Valestsky, P.; Appl. Catal., A 1999, 176, 75 .

24. Beller, M.; Fisher, H.; Kühlein, K.; Reisinger, C. P.; Herrmann, W. A.; J. Organomet. Chem. 1996, 520, 257.

25. Boury, B.; Corriu, R. J. P.; Adv. Mater. 2000, 12, 989.
26. Guimarães, A. P.; Rev. Bras. Ens. Fís. 2000, 22, 3.

27. Wohlfarth, E. P.; Fine Particle Magnetism, Springer Verlag: Berlin, 1986.

28. Kronmuller, H.; Micromagnetic Background of Hard Magnetic Materials, Kluwer Academic: Amsterdam, 1990.

29. Zanetti, S. M.; Dissertação de Mestrado, Universidade Federal de São Carlos, Brasil, 1997.

30. Chu, C.; Dunn, B.; J. Am. Ceram. Soc. 1987, 70, 375.

31. Semikolenov, V. A.; Llyna, I. I.; Simakova, I. L.; Appl. Catal., A 2001, 211, 91.

32. Al-Bawab, A.; Friberg, E. S.; Zuman, P.; Sjoblom J.; J. Dispersion Sci. Technol. 1998, 19, 571 .

33. Nijdam, A. J.; van Veenendaal, E.; Gardeniers, J. G. E.; Kentgens, A. P. M.; Nachtegaal, G. H.; Elwenspoeck, E.; J. Electrochem. Soc. 2000, 147, 2195 .

34. Shea, K. J.; Loy, D. A.; MRS Bull. 2001, 27, 368.

35. Klug, H.; Alexander, L.; X-Ray Diffration Procedures, John Willey and Sons: New York, 1954.

36. Nuffield, E. W.; X-Ray Diffration Methods, John Willey and Sons: New York, 1986.

37. Williams, D. B.; Carter, C. B.; Trasmission Eletron Microscopy III, Plenum Press: New York, 1996.

38. Leite, E. R.; Santos, L. P. S.; Carreño, N. L. V.; Longo, E.; Paskocimas, C. A.; Varela, J. A.; Lanciotti, F.; Campos, C. E. M.; Pizani, P. S.; Appl. Phys. Lett. 2001, 15, 78.

39. Barret, E. P.; Joyner, L. G.; Halenda, P. P.; J. Am. Chem. Soc. 1951, 73, 373.

40. Yanyong, L.; Takashi, H.; Ishii, T.; Kumagai, M.; Yasuda, H.; Suzuki, K.; Hamakawa, S.; Murata, K.; App. Catal., A 2001, $210,301$.

41. Volzone, C.; Masini, O.; Comelli, N. A.; Grzona, L. M.; Ponzi, E. N.; Ponzi, M. I.; Appl. Catal., A 2001, 214, 213.

42. Mahendran, M.; Iyakutti, K.; Scr. Mater. 2000, 42, 715.

43. Rodrigues-López, J. L.; Aguilera-Granja, F.; Vega, A.; Alonso, J. A.; Solid State Commun. 2000, 116, 309. 